Sonic Scope: New Approaches to Audiovisual Culture •

\title{
Windows into the Past - Raymond Sookram
}

\section{Raymond Sookram}

Published on: Oct 29, 2021

License: Creative Commons Attribution 4.0 International License (CC-BY 4.0). 


\section{Windows into the Past}

Raymond Sookram, Goldsmiths, University of London

A simple push of a mechanical button on an old PC tower triggers numerous whirs and beeps. Humming fills the void whilst Microsoft' $s$ Windows 95 logo and fluffy clouds appear on-screen. Then custom wallpaper. Then a deeply blissful performance.

Visit the web version of this article to view interactive content.

Video 1. Example of the Start-Up sequence for Windows 95, including “The Microsoft Sound" (0:54 - 1:00). MichaelComputerBoy, "Windows 95 Startup in the Year 2020," May 9, 2020, https://www.youtube.com/watch?v=XV3LdxpMzlc.

"Inspiring, universal ... optimistic, futuristic, sentimental, emotional" - just some of the adjectives Microsoft employed to guide composer and producer Brian Eno in crafting "The Microsoft Sound," for many an emblem of the late 1990s soundscape. 1 Simply hearing this tiny piece of music, lasting barely six seconds, evokes strong memories for childhoods and calmed anxieties of using a home PC for the first time. Now fast-forward to June 2021. Microsoft releases slowed down versions of multiple Windows start-up sounds, including "The Microsoft Sound," as a teaser for their upcoming Windows 11 operating system:

Visit the web version of this article to view interactive content.

Video 2. Microsoft's now unlisted video teasing the release of Windows 11. Windows, "Windows | Startup Sounds - Slo-fi Remix," June 10, 2021, https://www.youtube.com/watch?v=fMr4Qm5ZWrl\&t=1s. 
Note the opening on-screen caption: "Start relaxing with Windows Startup Sounds Slowed down 4,000\%." Or the video description: "Take a slow trip down memory lane with the Windows 95, XP, and 7 startup sounds slowed down to a meditative 4,000\% reduced speed." I am not sure why 4,000\% is "meditative," but I feel weirdly relaxed. I see my own memories of pushing the computer button as a three-year-old and hearing that start-up sound - and the people who were with me then now gone.

Vaporwave. Hauntology. Obsessions with imagined pasts. Of "the way things were." Music that promises an escape from the traumas of today - COVID, recessions, troubled politics - and a visual identity soaked in warm, beautiful nostalgia. Or as Dr Jennifer Walshe alludes to - the "synths, magenta grids and Greco-Roman statuary" of Floral Shoppe, complete with New York' s Twin Towers still in existence on its album cover. $\underline{2}$

We only need to look to the past few years to foreshadow the future of this audiovisual lulling. Towards animated cartoons enveloping the existential (or not); a British political party placing their Prime Minister as the reassuring protagonist of a Vaporwave "lo fi boriswave" video; or America's 45th President as its own central figure in

"Trumpwave" videos. $\underline{3}$ In tomorrow' s nostalgia, the new is not necessary - only memorable, audiovisual bliss is desired.

\section{Bibliography}

Alexander, Julia. "Sad cartoons and melancholic hip-hop inspired YouTube's new vaporwave scene." Polygon, September 6, 2018. https://www.polygon.com/2018/9/6/17823080/vaporwave-youtube-animation-musicchillhop.

Bullock, Penn and Eli Kerry., "Trumpwave and Fashwave Are Just the Latest Disturbing Examples of the Far-Right Appropriating Electronic Music.” Vice, January 30, 2017. https://www.vice.com/en/article/mgwk7b/trumpwave-fashwave-far-rightappropriation-vaporwave-synthwave. 
Selving, Joel. "Q and A With Brian Eno." SFGate, February 3, 2012. https://www.sfgate.com/default/article/Q-and-A-With-Brian-Eno-2979740.php.

Stokel-Walker, Chris. "lo fi boriswave: Why are the Conservatives posting 71-minute hypnotic videos to YouTube?” New Statesman, November 26, 2019. https://www.newstatesman.com/science-tech/2019/11/lo-fi-boriswave-why-areconservatives-posting-71-minute-hypnotic-videos-youtube.

Vektroid. Floral Shoppe. December 9, 2011. https://vektroid.bandcamp.com/album/floralshoppe.

\section{Media Cited}

MichaelComputerBoy. "Windows 95 Startup in the Year 2020." YouTube video, 1:09. May 9, 2020. https://www.youtube.com/watch?v=XV3LdxpMzIc.

Windows, "Windows | Startup Sounds - Slo-fi Remix." YouTube video, 11:00. June 10, 2021. https://www.youtube.com/watch?v=fMr4Qm5ZWrI\&t=1s.

\section{Footnotes}

1. Joel Selving, "Q and A With Brian Eno," SFGate, February 3, 2012, https://www.sfgate.com/default/article/Q-and-A-With-Brian-Eno-2979740.php...

2. Vektroid, Floral Shoppe, December 9, 2011, https://vektroid.bandcamp.com/album/floral-shoppe. 3. 
Julia Alexander, "Sad cartoons and melancholic hip-hop inspired YouTube' s new vaporwave scene," Polygon, September 6, 2018, https://www.polygon.com/2018/9/6/17823080/vaporwave-youtube-animation-musicchillhop;

Chris Stokel-Walker, "lo fi boriswave: Why are the Conservatives posting 71-minute hypnotic videos to YouTube?” New Statesman, November 26, 2019, https://www.newstatesman.com/science-tech/2019/11/lo-fi-boriswave-why-areconservatives-posting-71-minute-hypnotic-videos-youtube;

Penn Bullock and Eli Kerry, "Trumpwave and Fashwave Are Just the Latest Disturbing Examples of the Far-Right Appropriating Electronic Music," Vice, January 30, 2017, https://www.vice.com/en/article/mgwk7b/trumpwave-fashwave-far-right-appropriationvaporwave-synthwave. 